\title{
Descripción Histológica e Histoquímica del Hígado de Cobayo (Cavia porcellus)
}

\author{
Histological and Histochemical Description of the Liver of the Guinea Pig (Cavia porcellus)
}

\author{
"Carlos Rosas C.; ** Bélgica Vásquez P. \& ****Mariano del Sol
}

\begin{abstract}
ROSAS, C. C.; VÁSQUEZ, B. \& DEL SOL, M. Descripción histológica e histoquímica del hígado de cobayo (Cavia porcellus). Int. J. Morphol., 28(1):151-156, 2010.

RESUMEN: Si bien se han realizado estudios en el hígado de variadas especies, hay pocas investigaciones en cobayo, un roedor de fácil manejo y usado como animal de experimentación. El objetivo de este estudio fue describir en el hígado de cobayo, algunos aspectos histológicos e histoquímicos, lo cual permitiría realizar estudios experimentales. Se utilizaron 7 cobayos (Cavia porcellus) machos, obtenidos del Bioterio de la Universidad de La Frontera, Temuco, Chile. Las muestras fueron fijadas en formalina tamponada y procesadas para su inclusión en paraplast. Se realizaron cortes seriados de $5 \mu \mathrm{m}$, los que fueron teñidos para estudios histológico e histoquímico. El hígado presentó una arquitectura lobulillar con límites poco definidos y espacios porta constituidos por estructuras vasculares y canaliculares biliares. Los cordones parenquimatosos estaban constituidos por hepatocitos de aspecto cuboide a poligonal que se disponían en forma radial desde la vena centro lobulillar. En las células hepáticas se demostró la presencia de glucógeno, mucinas neutras y hemosiderina. El diámetro medio del hepatocito fue de 17,25 $\mu \mathrm{m}$ y el del núcleo fue de 7,31 $\mu \mathrm{m}$. El hígado de cobayo presenta similitudes con otras especies; sin embargo, para determinar parámetros morfológicos, se deben considerar: la edad, las condiciones medioambientales, la alimentación, etc.
\end{abstract}

PALABRAS CLAVE: Cobayo, Hígado, Hepatocito.

\section{INTRODUCCIÓN}

El hígado es la glándula más grande del cuerpo humano y posee una diversidad de funciones que se relacionan entre sí, tales como hematopoyéticas, metabólicas, secretoras y excretoras, entre otras.

Anatómicamente, el hígado presenta diferencias cuando es comparado entre especies, tanto por su forma como por su posición; sin embargo, en todos los animales se localiza adyacente al diafragma, en el hipocondrio derecho (Frandson \& Spurgeon, 1995).

Según Stinson \& Calhoun (1994), desde el punto de vista histológico, el hígado no presenta discrepancia de consideración. Principalmente, está compuesto de lóbulos hepáticos, los cuales están revestidos de células mesoteliales del peritoneo visceral que cubre una fina capa de tejido conjuntivo. El interior del hígado está dividido en lobulillos hepáticos. El tejido conjuntivo de la cápsula penetra en los espacios interlobulillares dando sostén al sistema vascular y a los conductos biliares. Una fina red de fibras reticulares rodea las células y sinusoides.

Frandson \& Spurgeon relatan que en el Hombre y otras especies, existe menos tejido conjuntivo interlobulillar, lo que no permite distinguir con facilidad los lobulillos hepáticos.

Fawcett (1995) y Al-Motagabani (2006) señalan que la interpretación de lobulillo poligonal clásico como la verdadera subunidad anatómica está apoyada por la observación que esta unidad está claramente delimitada por tabiques de tejidos conjuntivo en el hígado de cerdo ( $S u s$ scrofa), camello (Camelus dromedarios), oso (Ursus arctos), mapache (Procyon lotor) y ratas albinas (Rattus norvegicus). Junqueira \& Carneiro (1996) reafirman también la presencia de lobulillos hepáticos en diferentes especies y que en el Hombre no existen límites definidos de estas estructuras.

* Laboratorio de Embriología Experimental y Molecular, Programa de Anatomía y Biología del Desarrollo, Facultad de Medicina, Universidad de Chile, Chile.

** Universidad Autónoma de Chile, Sede Temuco, Chile.

${ }_{* * *}^{*}$ Facultad de Medicina, Universidad de La Frontera, Chile. 
Respecto a las columnas hepáticas, McLelland (1982) señala que en la gallina están formados por dos cordones de células hepáticas rodeadas por sinusoides. Similares observaciones realizaron Vicentini et al. (2005) en hígado de tilapia nilótica (Oreochromis niloticus) y Al-Motagabani en el de ratas albinas (Rattus norvegicus).

Respecto al hígado de cobayo (Cavia porcellus) Bhattacharjya et al. (2003) describieron que está compuesto de lobulillos hexagonales u ovalados, incompletos y separados por tejido conjuntivo, los que miden $684 \pm 19,28 \mu \mathrm{m}$ de diámetro. Señalaron además, que los hepatocitos son células redondas o alongadas que poseen núcleo central ubicados en forma de cordones. Por su parte, Rosas et al. (2007) señalaron, en base a estudios estereológicos, que la densidad de número de células hepáticas en este animal fue de 9,20 x 105 $/ \mathrm{mm}^{3}$, correspondiendo a una densidad de volumen de 70,04\% y densidad de superficie de $254,95 \mathrm{~mm}^{2} / \mathrm{mm}^{3}$. Agregaron además, que el número total de hepatocitos era de $2,8 \times 10^{7}$ y el de sinusoides $0,61 \times 10^{7}$.

El objetivo del estudio, fue determinar algunos aspectos histológicos e histoquímicos, así como el diámetro medio de la célula hepática y de su núcleo, en el cobayo (Cavia porcellus). Con la generación de estos nuevos conocimientos será posible sentar las bases para futuras investigaciones morfofuncionales.

\section{MATERIAL Y MÉTODO}

Se utilizaron 7 cobayos (Cavia porcellus) machos, adultos, clínicamente sanos y mantenidos con pellets y frutas ad limitum en el Bioterio de la Universidad de La Frontera, Temuco, Chile. Estos animales fueron sacrificados por traumatismo encéfalo craneano y, posteriormente, se le administró éter dietílico marca Merck $\mathrm{KGaA} \circledast$. A través de laparoscopía se procedió a la remoción del hígado, el que fue fijado en formalina tamponada al $10 \%$ a pH 7,4 por 36 horas. De cada hígado se seleccionó un trozo que fuese representativo del órgano.

Una vez fijadas las muestras, se procedió a realizar el protocolo de deshidratación, aclaración e inclusión en paraplast Tissue Prepâ marca Fisher Scientific para muestras histológicas. Obtenidos los tacos, los cortes se realizaron en un micrótomo rotatorio marca Micromâ HM325, con un grosor de $5 \mu \mathrm{m}$ de grosor y luego, fueron montados en portaobjetos. Los cortes de tejido desparafinados e hidratados, fueron teñidos siguiendo métodos adecuados para su observación histológica y análisis histoquímico.
Para el estudio histológico se realizaron las tinciones de hematoxilina-Eosina (HE), Tricrómico de Masson, Van Gieson y reticulina de Gomori. Para el análisis histoquímico, las secciones fueron teñidas con ácido peryódico de Schiff (PAS), azul Alcian (AA) al 1\%, pH 2,5 y azul de Prusia (AP). El estudio morfológico y las fotografías se realizaron con un microscopio óptico marca Olympusâ, modelo CX31, con cámara marca Moticamâ, modelo 480.

La determinación del diámetro medio del hepatocito y del núcleo celular se realizó observando 100 hepatocitos de forma aleatoria en los cortes histológicos de 5 hígados. Luego se calculó la desviación estándar (D.E.), el coeficiente de variación (C.V.), el error estándar (E.E.) y el coeficiente de error (C.E.).

\section{RESULTADOS}

Histológicamente, el hígado de cobayo (Cavia pocellus) presentó una arquitectura lobulillar y espacios porta constituidos por estructuras vasculares y canaliculares biliares. Los lobulillos hepáticos no presentaron una forma poligonal característica y sus límites no estaban bien definidos. En estas estructuras se observaron cordones de una sola célula hepática, que formaban columnas rodeadas por sinusoides vasculares de orientación radial, las cuales se dirigían a la vena centro lobulillar (Fig. 1). Estas venas formaban, generalmente, espacios ópticamente vacíos en los que con dificultad se observó su revestimiento endotelial. Los cordones parenquimatosos estaban constituidos por hepatocitos de aspecto cuboide a poligonal con un núcleo redondeado de cromatina laxa.

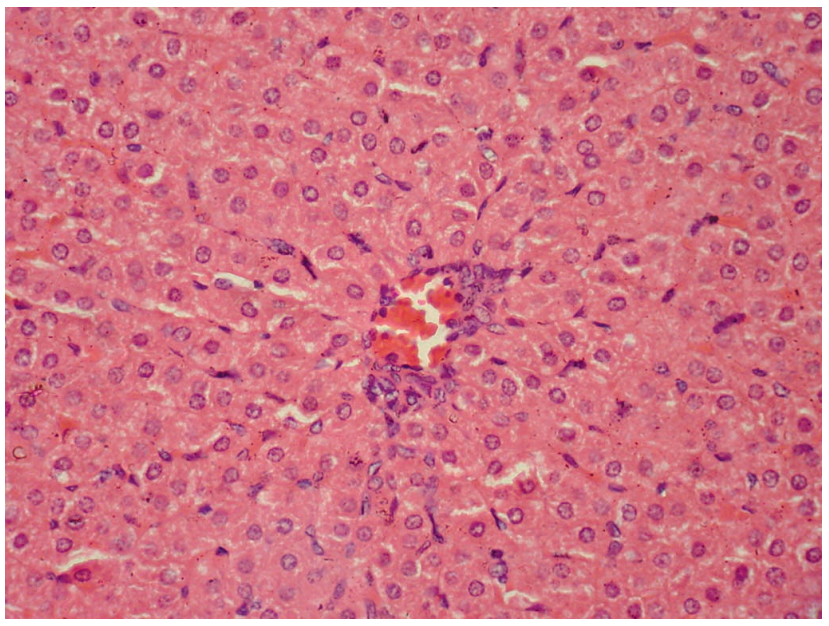

Fig. 1. Se observa la vena centro lobulillar en el hígado de cobayo (Cavia porcellus) con algunos glóbulos rojos y hepatocitos dispuestos en forma de cordones radiales rodeados por sinusoides vasculares. HE. (400X). 
El espacio porta presentaba una trama de tejido conjuntivo laxo, en la cual se apreciaron estructuras vasculares con pared muscular, correspondientes a las ramas de la arteria hepática, estructuras venosas de la rama de la vena porta y conductos biliares tapizados por epitelio cúbico del sistema colangiolar biliar (Fig. 2).

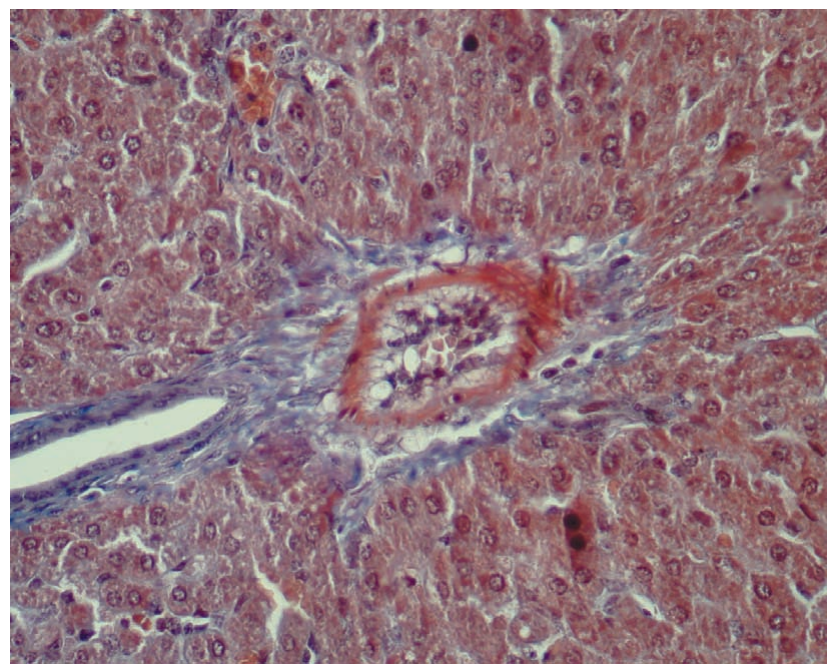

Fig. 2. Rama de la arteria hepática con células musculares (rojo) y fibras colágenas en la túnica adventicia (azul). Se observa también un conducto biliar tapizado con epitelio simple cúbico. Tricrómico de Masson. (400X).

El estudio histológico reveló también que las células hepáticas presentaban un núcleo basófilo y citoplasma eosinófilo, pudiendo observarse, en algunos casos, células binucleadas. La túnica adventicia de los vasos sanguíneos hepáticos presentaba fibras colágenas (Fig. 3) y gran cantidad de fibras reticulares distribuidas entre los cordones hepáticos (Fig. 4).

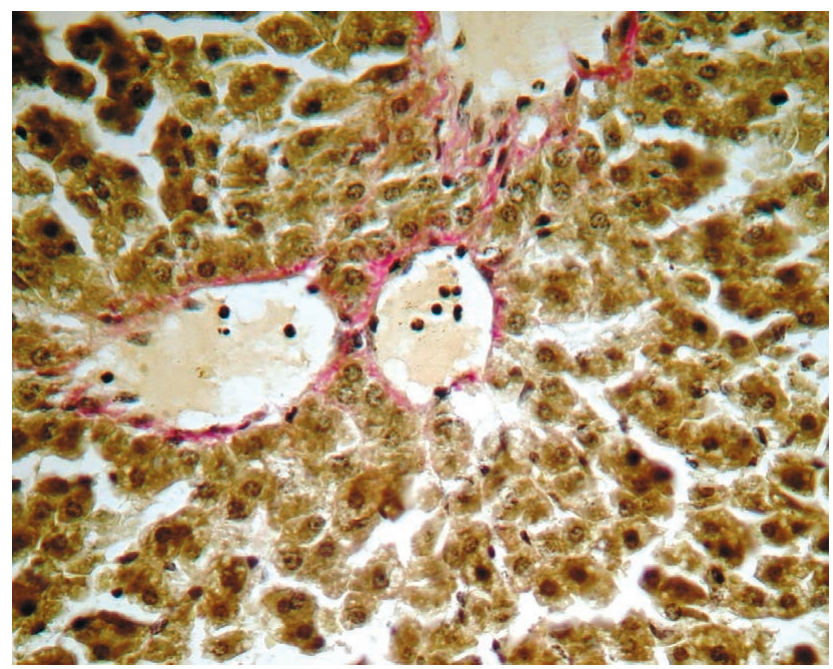

Fig. 3. Venas del lobulillo hepático de cobayo (Cavia porcellus). Fibras colágenas de la túnica adventicia (rojo). Van Gieson. (400X).

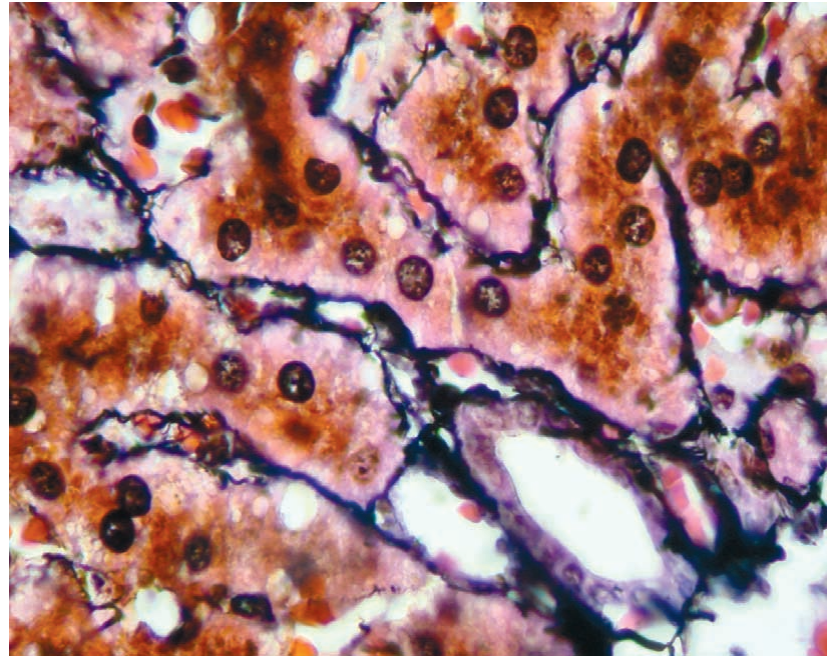

Fig. 4. Fibras reticulares (negro) formando una delgada malla alrededor de las células hepáticas. Reticulina de Gomori. (1000X).

Los resultados obtenidos con el métodos de PAS revelaron la presencia de glucógeno y mucinas neutras en todas los casos estudiados. Las células hepáticas mostraron un patrón de tinción variable, ya que se observaron hepatocitos de coloración púrpura de aspecto granulado y otros con aspecto espumoso y difuso, siendo este último patrón el que se presentaba con mayor frecuencia (Fig. 5). Las células hepáticas no mostraron una reacción alcianofílica para la tinción de AA al 1\%, pH 2,5, sólo se observó la tonalidad característica de la tinción de contraste usada (Fig. 6). Por otro lado, la tinción de AP demostró una baja presencia de depósitos de hemosiderina, encontrándose de forma aislada en el corte histológico (Fig. $7)$.

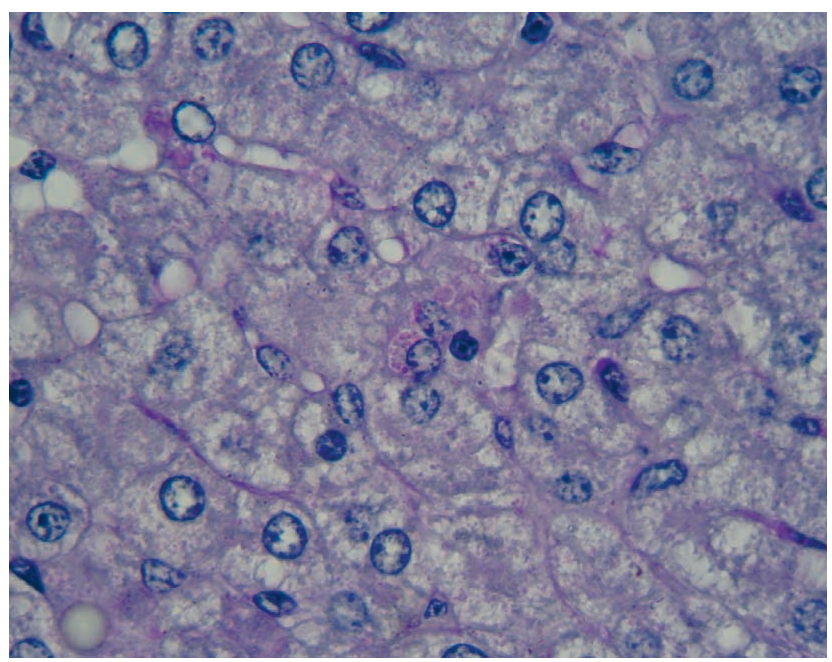

Fig. 5. Reacción púrpura de aspecto granulado positiva para glucógeno y mucinas neutras, en células hepáticas de cobayo ( $\mathrm{Ca}$ via porcellus). PAS. (1000X). 


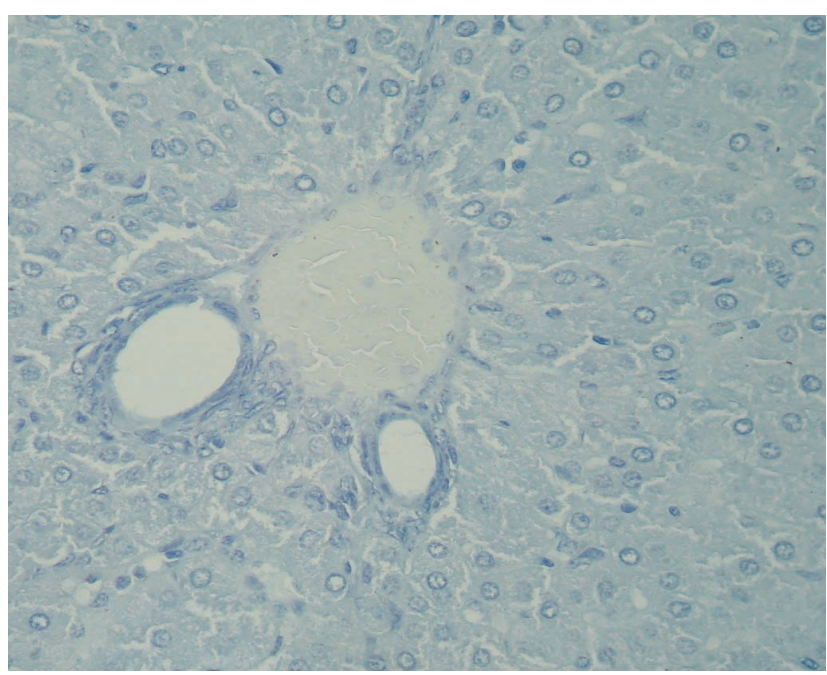

Fig. 6. Reacción negativa para mucopolisacáridos ácidos en células hepáticas de cobayo (Cavia porcellus). AA al 1\%, pH 2,5. (400X).

Los resultados obtenidos a través de la determinación del diámetro medio del hepatocito y del núcleo celular demostró que si bien poseen tamaños y formas variables, éstas no fueron significativas. Así, el diámetro medio del hepatocito fue de 17,25 $\mu \mathrm{m}$, con una D.E. de 2,68, un C.V. de $15,2 \%$, un E.E. de 1,2 y un C.E. de 6,93\%. El diámetro medio del hepatocito osciló entre 10,8 $\mu \mathrm{m}$ como mínimo y $27,0 \mu \mathrm{m}$ como máximo. El diámetro medio del núcleo hepático fue de $7,31 \mu \mathrm{m}$, con una D.E. de 0,94, un C.V. de $12,83 \%$, un E.E. de 0,42 y un C.E. de $5,73 \%$, siendo el diámetro mínimo de 5,94 $\mu \mathrm{m}$ y el máximo de 10,8 $\mu \mathrm{m}$.

\section{DISCUSIÓN}

En el cobayo (Cavia porcellus) el hígado presenta diferencias significativas en cuanto a tamaño, número de lóbulos y relación con órganos vecinos en comparación con el hombre (Rouvière \& Delmas, 2002) y otras especies, tales como tilapia nilótica (Oreochrmis niloticus) (Vicentini et al.) y coipo (Myocastor coypus) (Pérez \& Lima, 2007).

$\mathrm{Al}$ igual que lo descrito por Illanes et al. (2006) en el hígado de avestruz (Struthio camelus) y Bhattacharjya et al. en el hígado del cobayo (Cavia porcellus), observamos que la arquitectura histológica básica resulta similar al de otros animales en aspectos como, sinusoides, hepatocitos en columnas, venas centrolobulillares, espacio porta, ramas de la vena porta y arteria hepática y conductos biliares. Sin embargo, no presenta una distribución lobulillar con forma poligonal similar a un hexágono, observado en otros mamíferos como el cerdo (Fawcett y

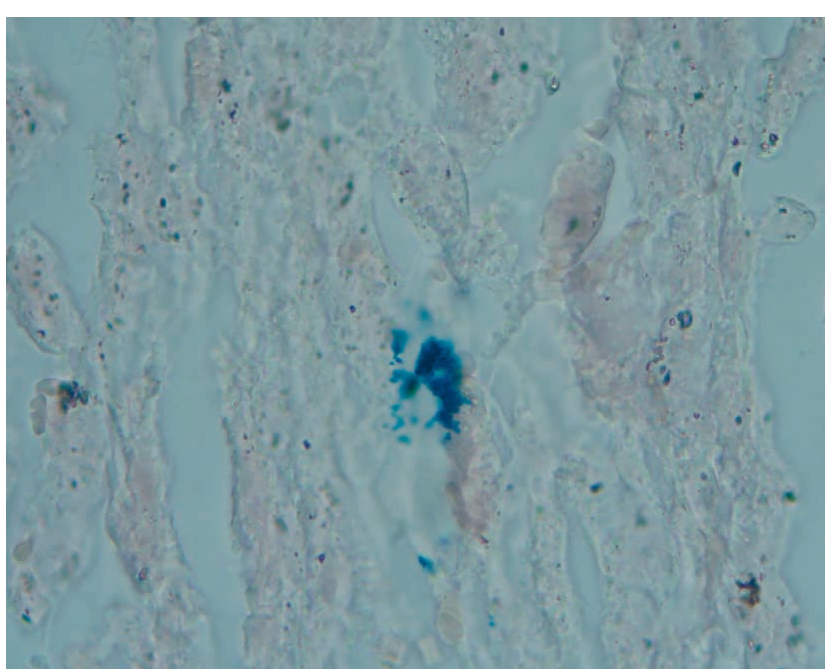

Fig. 7. Depósitos de hemosiderina en el citoplasma de células hepáticas de cobayo (Cavia porcellus). AP. (1000X).

Junqueira \& Carneiro) y ratas albinas (Al-Motagabani ), si no más bien límites poco definidos y que sólo se pueden asegurar cuando se está observando la vena centrolobulillar. Respecto al número de células que constituyen los cordones hepáticos, en el hígado de cobayo se observó que estaban constituidos por una célula, lo que difiere a lo señalado por McLelland en hígado de gallina y Vicentini et al. en hígado de tilapia nilótica, quienes describieron la presencia de dos células.

Por otro lado, es importante considerar el hecho que, aunque este órgano posea características histológicas similares, no necesariamente significa que su fisiología también lo sea, ya que el hepatocito al ser una célula multifuncional actúa de acuerdo a las necesidades del organismo. Es más, Stevens \& Lowe (2001) consideran que ni siquiera los hepatocitos de una misma especie son similares ya que, de acuerdo a su localización en el hígado, tendrán propiedades metabólicas diferentes.

Como lo señaló Crawford (2000), el glucógeno se encuentra en el citoplasma tanto de células músculo esqueléticas como de hepatocitos. Esta afirmación concuerda con nuestras observaciones en el hígado de cobayo. Sin embargo, el patrón de tinción no fue homogéneo en toda la lámina, pudiéndose observar una coloración púrpura con PAS de aspecto granulado, poco frecuente, hasta una coloración de aspecto espumoso y difuso, en la mayoría de los casos. Este hecho, puede atribuirse principalmente a la dieta de los animales, ya que según Cardell et al. (1973) y Fawcett existen diferencias sustanciales en la cantidad de glucógeno intracelular cuando los animales están expuestos a un ayuno prolongado. 
Otros componentes del metabolismo hepático han sido evaluados, tales como hierro almacenado y gránulos de hemosiderina. En el hígado de cobayo, los niveles de hemosiderina observados fueron escasos. Crawford considera que, en condiciones normales, pueden observase pequeñas cantidades de hemosiderina en médula ósea, bazo e hígado y que un exceso de estos depósitos puede conllevar a una hemosiderosis o hemocromatosis y esta última provoca graves lesiones hepáticas.

El diámetro medio observado del hepatocito del hígado de cobayo fue de $17,25 \mu \mathrm{m}$, medidas similares a los resul- tados obtenidos en hígado de ratas por Weibel et al. (1969) los cuales fueron $17,7 \mu \mathrm{m}$ de diámetro. Por otra parte, Junqueira \& Carneiro determinaron que el diámetro del hepatocito oscila entre 20 y $30 \mu \mathrm{m}$, confirmando los resultados obtenidos por otros autores. Sin embargo, existen diferencias en estos valores obtenidos en otras investigaciones, tales como las de Martins et al. (2005) en hígado fetal de ratas, quienes determinaron que el diámetro medio de estas células correspondía a 9,94 $\mu \mathrm{m}$. Estas diferencias podrían deberse a factores como la edad, condiciones medioambientales de los animales, diversidad de técnicas que existen para determinar parámetros morfológicos, entre otras.

ROSAS, C. C.; VÁSQUEZ, B. \& DEL SOL, M. Histological and histochemical description of the liver of the guinea pig (Cavia porcellus). Int. J. Morphol., 28(1):151-156, 2010.

SUMMARY: While studies have been conducted in the liver of different species, there is little research on the guinea pig, a rodent of easy handling and used as an experimentation animal. The aim was to describe in the guinea pig liver, some histological and histochemical aspects, which would allow experimental studies to be carried out. We used seven male guinea pigs (Cavia porcellus) obtained from the Bioterium of the Universidad de La Frontera, Temuco, Chile. Samples were fixed in buffered formalin and processed for inclusion in paraplast. Serial thick of $5 \mu \mathrm{m}$ were stained for histological and histochemical studies. The liver presented a lobular architecture vague borders and portal tracts consisting of vascular structures and canalicular bile. Parenchymal cords were composed of hepatocytes with a cuboidal to polygonal appearence, and radially distributed from the central lobular vein. It showed the presence of glucogen liver cells, and hemosiderin neutral mucins. The average diameter of hepatocytes was $17.25 \mu \mathrm{m}$ and the nucleous was of $7.31 \mu \mathrm{m}$. The guinea pig liver shows similarities with other species, however, to determine morphological parameters, age, environmental conditions of animals, feeding, etc. must be considered.

KEYWORDS: Guinea pig; Liver; Hepatocyte.

\section{REFERENCIAS BIBLIOGRÁFICAS}

Al-Motagabani, M. A. Histological and histochemical studies on the effects of methotrexate on the liver of adult male albino rat. Int. J. Morphol., 24(3):417-22, 2006.

Bhattacharjya, M. K.; Surendrajit, K. \& Banerjee, R. Histological, biometrical and lobar pattern of liver in guinea pig. GEOBIOS, 30:265-7, 2003.

Cardell, R. R.; Larner, J. \& Babcock, M. B. Correlation between structure and glycogen content of livers from rats on a controlled feeding schedule. Anat Rec., 177(1):23-37, 1973.

Crawford, J. M. El Hígado y las vías biliares. In. Cotran, R. S.; Kumar, V. \& Collins T. Robbins: Patología Estructural y Funcional. $6^{a}$ ed. Madrid, Mc Graw-Hill, 2000. pp. 881-940

Fawcett, D. W. Hígado y Vesícula Biliar. In. Bloom-Fawcett. Tratado de Histología. $12^{\circ}$ ed. Madrid, Interamericana-Mc Graw Hill, 1995. pp 713-48.

Frandson, R. D. \& Spurgeon, T. L. Anatomía del Aparato Digestivo. Anatomía y Fisiología de los Animales Domésticos. México, Editorial Interamericana-Mc. Graw Hil, 1995. pp 323-4.

Illanes, J.; Fertilio, B.; Quijada, M.; Leyton, V. \& Verdugo, F. Des- cripción histológica de las glándulas anexas del aparato digestivo (Struthio camelus var. domesticus) Int. J. Morphol., 24(3):297-302, 2006.

Junqueira, L. C. \& Carneiro J. Histología Básica Texto y Atlas. 4 ed. Madrid, Masson, 1996. pp.306-19.

Martins, A. T.; Azoubel, R.; Lopes, R. A.; Di Matteo, M. A. S. \& Arruda, J. G. F. Effect of sodium cyclamate on the rat fetal liver: A karyometric and stereological study. Int. J. Morphol.,23(3):221-6, 2005.

McLelland, J. Sistema digestivo de las aves. In. Sisson, S. \& Grossman J. D. Anatomía de los Animales Domésticos. Barcelona, Salvat Editores, 1982. Tomo II. pp. 2059-60.

NRC (National Research Council) Guide for the Care and Use of Laboratory Animals. Washington, DC. National Academy of Science. 1996.

Pérez, W. \& Lima, M. Anatomical description of the liver, hepatic ligaments and omenta in the coypu (Myocastor coypus). Int. J. Morphol., 25(1):61-4, 2007.

Rosas, C. C.; Vásquez, B \& del Sol, M. Estudio estereológico del 
hígado de cobayo (Cavia porcellus). Int. J. Morphol., 25(3):557-560, 2007.

Rouvière, H. \& Delmas, A. Hígado y vías biliares. Anatomía humana descriptiva, topográfica y funcional. Barcelona, Masson, 2002. Tomo II. pp.388-412.

Stevens, A. \& Lowe, J. Hígado, vías biliares y páncreas. Anatomía Patológica. $2^{\mathrm{a}}$ ed. Madrid, Ediciones Harcourt. 2001. pp 273-9.

Stinson, A. W. \& Calhoun, M. L. Sistema Digestivo. Dellmann, D. H. Histología Veterinaria. Zaragoza, Editorial Acribia, 1994. Pp. 213-7.

Vicentini, C. A.; Franceschini-Vicentini, I. B.; Bombonato, M. T. S.; Bertolucci, B.; Lima, S. G. \& Santos, A. S. Morphological study of the liver in the teleost Oreochromis niloticus. Int. J. Morphol., 23(3): 211-5, 2005.

Weibel, E. R.; Staubli W.; Gnagi H. R. \& Hess F. A. Correlated morphometric and biochemical studies on the liver cell - I. Morphometric model, stereologic methods, and normal morphometric data for rat liver. J. Cell Biol., 42:68-91, 1969.
Correspondencia a: Carlos Rosas C.

Laboratorio de Embriología Experimental y Molecular Facultad de Medicina

Universidad de Chile

Av. Independencia 1027

Santiago - CHILE

Email: crosasc@med.uchile.cl

Recibido : 22-12-2009

Aceptado: 18-01-2010 